\title{
FECUNDIDAD Y ACTIVIDAD FEMENINA
}

\section{Graciela Sarrible}

Universidad de Barcelona

RESUMEN. La relación inversa entre fecundidad y actividad femenina ha constituido uno de los postulados de la Teoría de la Transición Demográfica y de las formulaciones corrientes. La participación de la mujer en la población activa en España no ha sido continuamente creciente, como a veces se estima, sino que depende de la coyuntura económica y de contextos sociales específicos. Han existido situaciones de mayor participación en el pasado, que han cambiado. Este decrecimiento desdice la formulación general enunciada, al menos en el corto plazo. Frente a esta situación fluctuante, el descenso de la fecundidad en la última década ha sido significativo. En esta medida, no se puede establecer una correlación entre actividad femenina remunerada y fecundidad, en plazos relativamente cortos. Las sociedades europeas no tienen la misma evolución. Un estudio comparativo se impone. En este trabajo se analiza la situación de la actividad femenina y de la fecundidad en España, Francia, Italia y la RFA, a partir de los datos de los Anuarios de la OIT y de la serie Eurostat.

\section{INTRODUCCION}

Las razones por las cuales las mujeres deciden tener hijos resultan aún hoy, para el investigador, inciertas. De las magnitudes macroeconómicas y sociológicas de las grandes teorías se ha pasado a considerar los valores sociales (Vichnevskij, 1987). Esta aparente dualidad implicaría la alternancia de explicaciones excluyentes. Unas teorías se suceden a otras, por no resultar satisfactorias. Si el poder predictivo de estas formulaciones es reducido, su poder explicativo también lo es. 
Una de las últimas revisiones de la Teoría de la Transición Demográfica (Chesnais, 1986) representó un intento de rescatar un marco más general para interpretar los cambios demográficos. El hecho de haberlo conseguido es discutible. Sin embargo, el intento no es vano. Si recuperamos la riqueza explicativa de las teorías del siglo XIX, no se partirá de cero. Desde la biología hasta la economía, estas formulaciones tenían un carácter global e integrador que muchas de las últimas no poseen. En la medida en que la Sociología no se había consolidado como ciencia, las variables sociales eran tenidas en cuenta por todos los investigadores, independientemente de su formación (Overbeek, 1974). Las formulaciones que se pretenden novedosas (por ej., Caldwell, 1982) no hacen más que redefinir otras del siglo XIX, cuyo antecedente no mencionan o desconocen.

Todo este cuestionamiento lleva a replantearse no sólo la limitación de los marcos teóricos que intentan explicar la evolución de la fecundidad, sino también su validez. Pensar que los condicionantes que pueden influir en el número y momento de tener la descendencia pueden ser los mismos que los que surgieron de una sociedad que llevaba a cabo la revolución industrial y la demográfica, es negar la esencia del cambio social. La sociedad de finales del milenio tiene otras prioridades y otros valores, que se dejan traslucir en los comportamientos de la esfera privada, como la familia.

La sociedad europea occidental y oriental es muy diferente a aquella que le dio origen a través de una revolución burguesa. No se trata de una mera alteración cuantitativa en el número. Las parejas que se constituyen en la actualidad son diferentes y no repiten esquemas anteriores. Al mismo tiempo, un proceso de homogeneización permite semejanzas entre lo que sucede en el conjunto de Europa. Si antes las diferencias entre las sociedades eran fundamentales para entender los estadios de la evolución en que se encontraban, hoy resulta difícil hablar de etapas y de inscripción en ellas. La rapidez de algunos cambios, como los sucedidos en España en la década de los ochenta, limita la comparación con otras sociedades para poder hacer previsiones, pero permite establecer paralelos en las actitudes de los jóvenes.

El marcado descenso de la fecundidad en España no puede ser explicado por una relación directa y exclusiva con las mismas variables que antes coexistían con una tasa de reproducción por encima de los niveles de reemplazo. Esta reducción no es meramente coyuntural. Es improbable que se vuelva a los niveles registrados con anterioridad. Tampoco resulta del todo factible que este descenso sea permanente. Estos cambios que está registrando la sociedad española han ocurrido en otras sociedades europeas con mayor lentitud. La respuesta del gobierno ha sido de sorpresa y de temor. De sorpresa, porque la transformación ha sido muy rápida, al registrarse en un corto espacio de tiempo. De temor, porque se teme al envejecimiento o a la reducción de la población, como si ello fuera un mal en sí mismo. 
En este contexto no se pueden copiar ni repetir experiencias ajenas. Las medidas de política familiar tomadas en otros países responden a realidades diferentes y a procesos más lentos. El efecto de las ayudas familiares se ha mostrado limitado. No es evidente que se alcancen los objetivos de manera inmediata. Además, los gobiernos deben tener bien claro lo que pretenden, sobre todo a nivel de un determinado marco valorativo. Las políticas de sostén económico pueden tener efectos perversos y no contemplados. Es necesario tener conciencia clara de los objetivos; saber si sólo se busca el incremento del número o si se trata de favorecer núcleos familiares alternativos al tradicional.

Definir una política implica conocer la sociedad a la que va destinada. No se pueden tomar medidas arbitrariamente, sin estimar sus consecuencias sociales. Actuar en determinadas esferas implica una voluntad inequívoca de alterar ciertas tendencias que se consideran insatisfactorias. Las medidas tomadas en Francia para retirar a la mujer del mercado de trabajo constituyen un buen ejemplo. La reproducción social descansa en el salario que se le paga a una mujer, futura madre de un tercer hijo, por abandonar un empleo y permanecer en la casa para cuidarlo. Estas acciones definen un contexto teórico e ideológico específico. Por una parte, se estima que la influencia del empleo es negativa para una probabilidad de agrandamiento de $a_{2}$. O sea, que el trabajo de la mujer influiría en el incremento de la descendencia más allá del segundo hijo. Por otra parte, el Estado considera lícito plantear este enfrentamiento entre maternidad y trabajo. El marco valorativo que esta decisión implica jerarquiza la crianza de los hijos y la actividad remunerada de la mujer, al mismo tiempo que plantea una oposición irresoluble entre ellas.

En el caso de España, sería necesario considerar la relación que se establece entre estas dos variables. No tiene que ser necesariamente la misma que en las restantes sociedades europeas. Primero, porque la evolución de la participación femenina no ha sido similar. En España se han registrado oscilaciones en la entrada y salida de las mujeres de la población activa. Segundo, porque todavía no se han alcanzado los niveles de participación de las restantes sociedades. Tercero, porque todo ello implicaría que las motivaciones para trabajar puedan no ser las mismas. Si la población activa femenina española está sesgada, por ser un grupo reducido, las razones que le impelen a trabajar pueden ser de otra índole diferente que si se tratara de la mayoría de la población potencialmente activa.

Este artículo pretende estudiar la evolución de la actividad femenina en los últimos años y su relación con el descenso de la fecundidad. Esto implica caracterizar y destacar los rasgos particulares del proceso en España, por oposición a otros países europeos occidentales. La concomitancia de las transformaciones acaecidas a la población activa femenina española y el proceso de descenso brusco de la fecundidad podrían apuntar a una relectura de la relación de estas variables. Hasta qué punto el cambio de com- 
portamiento de los jóvenes no es permanente y las nuevas actitudes más individualistas no condicionan de una forma diferente los proyectos de vida de las mujeres y de su pareja; es un hecho que debe ser estudiado.

\section{ANTECEDENTES}

La relación entre actividad femenina y fecundidad ha sido tratada en el contexto de diversas sociedades. En algunos casos existe un cuestionamiento subyacente de la Teoría de la Transición Demográfica al quedar demostrado que la influencia no es directa ni lineal. Un hecho tan complejo como la constitución de la descendencia no puede ser reducido a condicionamientos económicos o macrosociales. La formulación en términos de causa-efecto implica la unidireccionalidad del proceso. Las determinaciones no pueden ser absolutas. La simplificación de la realidad no debe conducir a los investigadores al enunciado de fórmulas o modelos que prescindan de la riqueza de lo social.

Un estudio de las Naciones Unidas sobre los factores que afectan a la fecundidad estima que los económicos; sociales, demográficos y de salud son relevantes. Esta investigación se inscribe en la Teoría de la Transición Demográfica, como apéndice de la Teoría de la Modernización. La educación es considerada como un atributo del status socioeconómico y no como un factor per se. De acuerdo con el citado estudio, no es posible encontrar una relación estadística simple entre educación y fecundidad, sino tres modelos posibles. El primero, donde la influencia es negativa, sólo podría explicar una diferencia de 0,18 hijos, entre los extremos de los niveles de instrucción. El segundo es la curva en forma de "U", característica de Francia, Italia y Gran Bretaña, cuantificando las diferencias en niveles similares $(0,17)$. Bélgica y España configurarían el tercer modelo, atípico, donde no nodría establecerse una clara relación entre las dos variables (UN, 1983).

La influencia del trabajo femenino en la fecundidad está considerada como negativa, por hipótesis, independientemente del nivel de desarrollo de las sociedades que se analicen. La lejanía del trabajo y la posibilidad de contar con familiares que sustituyan a la madre son hechos tenidos en cuenta, pero tratados colateralmente respecto a la relación entre las dos variables. En los países occidentales esta influencia sería determinante, aun controlando otros factores. Los casos atípicos, donde las diferencias no pueden ser tenidas por estadísticamente significativas, son Polonia, Italia, España y Yugoslavia, según el citado estudio. La conclusión es que el trabajo de la mujer y la crianza de los hijos tienden a constituir roles incompatibles, en los países más desarrollados (UN, 1983).

$\mathrm{El}$ informe de las Naciones Unidas es un claro ejemplo de la generalización de un enunciado y de la marginalización de situaciones que no pueden ser explicadas en el marco teórico definido. España aparece citada como 
caso atípico en las dos variables mencionadas. Sin embargo, de acuerdo con los resultados de las dos Encuestas de Fecundidad que se realizaron en 1977 y 1985, la actividad y la fecundidad mantendrían esa relación negativa enunciada para las otras sociedades. Esto implica que es necesario estudiar cada caso en un marco temporal más amplio y no definir las variables en contextos ahistóricos. Los cortes diacrónicos permiten establecer comparaciones entre las sociedades, pero impiden verificar la evolución que ha tenido lugar.

En la sociedad yugoslava, el impacto de la educación y del empleo femenino parecen inscribirse en la clásica relación enunciada precedentemente. Pero es necesario introducir matices. Todorovic ha estudiado la evolución del tamaño de la familia, considerando simultáneamente las tendencias de las dos variables mencionadas. Estimó que la validez de la hipótesis podía circunscribirse a ciertos estadios de la Transición Demográfica. O sea, que su poder explicativo es válido, pero limitado a ciertas situaciones. Las diferencias entre las distintas categorías de las variables tienden a desaparecer paulatinamente o a atenuarse. El poder explicativo de las variables es mayor cuando más alta es la fecundidad (Todorovic, 1985). Ello implica que en las sociedades donde la fecundidad ha disminuido considerablemente, las relaciones con los factores que la influenciaban en una etapa anterior se han transformado.

Estudios realizados sobre el mercado de trabajo femenino en España matizan las relaciones entre las variables, sin hacer mención de an condicionamiento específico o predeterminación. La educación es el factor explicativo de los ingresos de la mujer que trabaja (Fernández, 1987). Al igual que los estudios realizados en Francia, la mutua interdependencia entre la capacitación obtenida a través del sistema educativo y la ocupación (Boudon, 1973) queda probada. La permanencia de la mujer en el trabajo depende del salario que ella gane. La posibilidad de ser reemplazada en su hogar está condicionada por su nivel económico. La relación entre trabajo y fecundidad, tal cual había sido definida en la Teoría de la Transición Demográfica, no ha podido ser corroborada (Fernández, 1987).

En términos generales, los estudios mencionados cuestionan subrepticiamente el efecto negativo entre trabajo y maternidad. Sin desmentir las formulaciones clásicas, éstas no pueden ser refrendadas en la sociedad contemporánea. En el próximo pasado, el modelo definido todavía tenía visos de verosimilitud. Pero cuando se alcanza un cierto estadio, que se corresponde con una tasa de fecundidad relativamente reducida, es imprescindible revisar los planteamientos anteriores. La generalización de un solo enunciado tiene que dar paso a la consideración de la situación por clase social. En términos teóricos, representa un retorno a las formulaciones del siglo XIX, que no dejaban de tener en cuenta las diferencias sociales como fundamentales en la explicación de los distintos hechos demográficos, pero sobre todo en los aspectos de la fecundidad diferencial (Coontz, 1960). 
La fecundidad acumulada de la mujer, o sea, su descendencia, depende de su categoría socioprofesional. La vivencia de la maternidad y la forma de resolver los conflictos que provoca dentro del hogar es muy diferente en la obrera, en la administrativa y en la profesional liberal. La creciente inserción de las mujeres con mayor nivel de instrucción en el mercado de trabajo no sólo es signo de un creciente nivel de capacitación, sino también de una forma de compatibilizar los roles de madre y trabajadora. Al mismo tiempo, las obreras salen progresivamente de la población activa. Tener en cuenta el proceso de evolución de la mujer en el mercado de trabajo es una de las claves para entender las alteraciones producidas en la fecundidad de la última década.

\section{LOS DATOS}

Para estudiar la evolución de la población activa femenina se han utilizado los datos del Anuario de Estadísticas del Trabajo de la OIT. La serie Eurostat contiene información sobre la población de los países europeos y sus características. El conjunto de estos datos publicados tienen un origen común. Son todas fuentes secundarias y oficiales. Los organismos internacionales publican las estadísticas que son remitidas por los propios gobiernos. Por ello, los años de referencia no siempre coinciden. Puede suceder que en una misma publicación se incluyan datos de distintos años.

Este estudio se centra en los años ochenta. Se trata de analizar las tendencias de las variables en la sociedad española, contrastándolas con la evolución que ha tenido lugar en otros países europeos. Las sociedades con las cuales se establecerán comparaciones son Francia, Italia y RFA. Han sido elegidas no sólo por los paralelos que habitualmente se establecen con otras sociedades mediterráneas o católicas, como Francia o Italia, sino también por la evolución que ha tenido la fecundidad en esas sociedades. En el caso de la RFA, lo ha sido por esta última razón y porque constituye un ejemplo de limitada y no explícita intervención política a nivel de la reproducción de la población.

Estudiar la evolución de la actividad femenina en el mercado de trabajo implica referirse a los niveles de participación, al peso de las mujeres en los puestos que exigen mayor capacitación y a su nivel de independencia respecto de la población masculina. Las tablas 1 a 3 contienen los indicadores calculados a partir de los datos publicados por la OIT en su Anuario de los años 1983 y 1985. Los datos de España han sido extraídos del mismo Anuaria que los restantes países europeos. Las tablas 4 y 5 contienen indicadores de la fecundidad en la presente década.

En la tabla 1 se refleja la proporción de mujeres en el total de la población activa para España, Francia, Italia y RFA, para los años 1982 y 1984 . El espacio de tiempo que media entre ambas fechas es muy corto. Sin embar- 
go, es indicativo de una lenta evolución en los restantes países europeos. Mientras que en España la participación de la mujer se ha reducido considerablemente, en los restantes países europeos la tendencia al alza es evidente en los años elegidos. Toda elección puede tacharse de arbitraria. Es evidente que los altibajos que ha tenido la población activa femenina en España en los últimos años no se han registrado con la misma intensidad en otros períodos de tiempo. En el caso de la fecundidad puede decirse lo mismo. Se insiste en esta década porque ha sido la de los cambios cruciales y relativamente sorprendentes.

Los niveles de participación femenina en Francia y RFA son similares. En un nivel medio se encuentra Italia. España tiene el menor porcentaje de mujeres en la población activa. En 1982 no llegaba al tercio, pero dos años más tarde apenas alcanza un cuarto. En los casos de Italia y España se debe señalar que, posiblemente, estos datos enmascaren una marcada participación en la economía sumergida. Las mujeres que salen de las estadísticas oficiales como trabajadoras bien pueden pasar, en determinadas circunstancias, a los trabajos no-declarados. Sin embargo, esta subestimación no alcanza a explicar las diferencias observadas. Incluso este error podría ser más significativo en Italia que en España. Es evidente que en la sociedad española se ha registrado un retroceso de la mujer en la población activa, en los inicios de la década de los ochenta, coincidiendo con la crisis económica. En una situación donde el desempleo crece es factible que la mujer se retire del mercado de trabajo. En circunstancias difíciles, la mujer no busca constantemente empleo, como en el caso de la población masculina, sino que espera otra coyuntura económica más favorable.

\section{TABLA 1}

Proporción de mujeres en la población activa (Varios países europeos, 1982 y 1984)

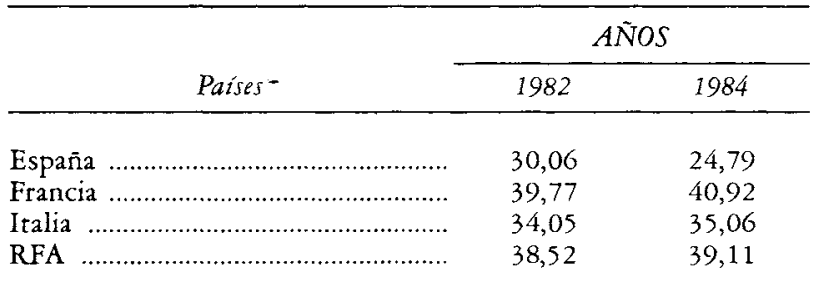

FuENTE: OIT, Anuario de Estadisticas del Trabajo, Ginebra, 1983 y 1985 . 
En la tabla 2 se ha calculado la proporción de profesionales y técnicos superiores respecto al total de la población activa de cada sexo y la que trabaja por cuenta propia respecto al total de su misma categoría. El primer hecho a destacar es que en todos los países la proporción de mujeres que pertenecen a esta categoría es superior a la de los hombres. Esto se debe a que la población femenina está sesgada. La mayoría de los hombres trabajan, por lo que la participación de los profesionales refleja exactamente su peso en el total de la población. En el caso de las mujeres, al tratarse de sólo una parte de la población potencialmente activa, la proporción de profesionales y directivos del sexo femenino es mayor. Ello implica que las mujeres que trabajan son las que tienen mayor capacitación, respecto al conjunto de mujeres que podrían hacerlo.

Las proporciones de profesionales de cada sexo en Francia y RFA resultan próximas entre las dos subpoblaciones y entre los dos países. Italia, en cambio, tiene una proporción muy baja, relativamente, de hombres en esta categoría, y alta entre las mujeres. Hay 65 por 100 más de mujeres en esa categoría que hombres. Las proporciones de cada sexo en España son próximas, como en Francia y en RFA, pero mucho más reducidas respecto al total de la población activa. Esto apunta a un menor nivel medio de instrucción en España, tanto en hombres como en mujeres.

Si analizamos la independencia de los profesionales, las diferencias entre hombres y mujeres son significativas. El proceso de salarización de la categoría superior alcanza y afecta en mayor medida a la población activa femenina. Tanto en España como en la RFA, hay 2,3 y 2,5 hombres por cada mujer trabajando por cuenta propia en esa categoría profesional. En cambio, en Italia, la proporción se eleva a 5,3 hombres por cada mujer. Dos fenómenos aparentemente contradictorios se manifiestan. Por una parte,

\section{TABLA 2}

Proporción de profesionales y técnicos superiores en el total de activos, distinguiendo los trabajadores por cuenta propia por sexo

(Varios países europeos)

\begin{tabular}{|c|c|c|c|c|}
\hline \multirow[b]{3}{*}{ Paises } & \multicolumn{4}{|c|}{ PROPORCIONES POR SEXO } \\
\hline & \multicolumn{2}{|c|}{ Profesionales/Total } & \multicolumn{2}{|c|}{ Cuenta propia } \\
\hline & $H$ & $M$ & $H$ & $M$ \\
\hline 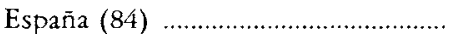 & 6,05 & 8,99 & 14,71 & 6,42 \\
\hline Francia $(82)$ & 13,87 & 14,51 & - & - \\
\hline 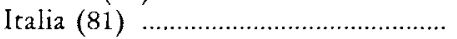 & 9,43 & 15,54 & 17,88 & 3,37 \\
\hline RFA (84) & 13,44 & 14,55 & 13,64 & 5,51 \\
\hline
\end{tabular}

Fuente: OIT, Anuario de Estadísticas del Trabajo, Ginebra, 1985. 
hay más mujeres profesionales trabajando que hombres. Pero estas mujeres están integradas en instituciones y dependen de otros. No son independientes. Al contrario que los hombres, que han logrado mantener la clásica independencia de los profesionales liberales. Las diferencias entre los sexos son mayores en Italia, en los dos hechos estudiados. La mayor proporción de mujeres profesionales implica un proceso de salarización más acentuado en esa categoría profesional para este sexo, pero no para los hombres, que evidencian mayor nivel de independencia, respecto a los restantes países. La integración de la mujer en el mercado de trabajo no le ha reportado una igualdad de condiciones respecto de los hombres, con su mismo nivel de capacitación.

En la tabla 3 se han calculado las proporciones de profesionales y trabajadoras por cuenta propia respecto a otra población de referencia. En la tabla 2 se medían estos porcentajes al interior de las respectivas subpoblaciones activas de cada sexo y de cada categoría. En la tabla 3 se ha estimado el peso de las mujeres profesionales respecto al total de la categoría (ambos sexos) y, por lo tanto, la feminización de este grupo. De la misma manera, se han calculado las trabajadoras por cuenta propia respecto al total de trabajadores por cuenta propia.

El peso de las mujeres profesionales y técnicos superiores en España es similar al de los restantes países. A pesar de ser el más reducido de todos, está próximo a los niveles de Francia y la RFA. Si comparamos estos resultados con los de la tabla 2, se debe señalar que si bien la mujer participa en una relación de 4 a 6 respecto a los hombres en esta categoría, los profesionales todavía tienen un peso reducido en el conjunto de la población activa, respecto a otros países. En la medida en que se continúe incrementando el

TABLA 3

Indicadores de feminización de la actividad. Proporción de mujeres profesionales y de mujeres trabajando por cuenta propia sobre el total (Varios países europeos)

\begin{tabular}{|c|c|c|}
\hline \multirow[b]{2}{*}{ Paises } & \multicolumn{2}{|c|}{ PROPORCION MUJERES } \\
\hline & $\begin{array}{c}\text { Profesionales } \\
\text { Total }\end{array}$ & $\begin{array}{c}\text { Cta. propial } \\
\text { Total }\end{array}$ \\
\hline España (84) & 39,05 & 21,69 \\
\hline Francia (82) & 41,40 & - \\
\hline Italia (84) & 46,34 & 14,00 \\
\hline RFA (84) & 41,01 & 21,95 \\
\hline
\end{tabular}

Fuente: OIT, Anuario de Estadisticas del Trabajo, Ginebra, 1985. 
nivel medio de instrucción de los españoles, este grupo social aumentará su peso en el conjunto.

El caso de Italia se ha mostrado atípico respecto a los otros elegidos. Francia y la RFA tienen indicadores de niveles próximos, mientras que Italia y España muestran situaciones extremas. Se diría que mientras el primero es un ejemplo de lo que puede ser la evolución futura de la población activa en Europa, España no ha alcanzado los niveles medios. En Italia, casi la mitad del grupo de profesionales son mujeres. Pero en este país, más que en ningún otro, esa proporción creciente de mujeres con elevada capacitación ha desembocado en una pérdida de independencia y en un proceso de salarización.

Si bien la tendencia de la población activa femenina es a incrementarse en los restantes países europeos, la fecundidad ha presentado oscilaciones durante el período. La tendencia secular posterior al Baby-Boom de la posguerra era a un descenso permanente, que situó a varios países europeos por debajo del reemplazo. La década de los ochenta ha sido de cambios. Esta tendencia a la baja se ha interrumpido en Francia y en la RFA. En Italia, en cambio, la fecundidad continúa disminuyendo. En la tabla 4 constan los Indices Sintéticos de Fecundidad correspondientes a los países estudiados desde 1980 en adelante.

TABLA 4

Indice Sintético de Fecundidad, 1980-1986

(Varios países europeos)

\begin{tabular}{|c|c|c|c|c|c|c|c|}
\hline \multirow[b]{2}{*}{ Paises } & \multicolumn{7}{|c|}{ AÑOS } \\
\hline & 1980 & 1981 & 1982 & 1983 & 1984 & 1985 & 1986 \\
\hline España & 2,22 & 2,03 & 1,94 & 1,79 & 1,72 & 1,64 & 1,54 \\
\hline 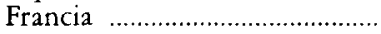 & 1,94 & 1,94 & 1,91 & 1,79 & 1,81 & 1,82 & 1,84 \\
\hline Italia & 1,69 & 1,62 & 1,59 & 1,52 & 1,46 & 1,41 & 1,32 \\
\hline RFA & 1,44 & 1,43 & 1,41 & 1,33 & 1,29 & 1,28 & 1,34 \\
\hline
\end{tabular}

NOTA: Los datos de Francia corresponden a la población metropolitana (territorio europeo).

Fuente: EUROSTAT, Estadísticas Demográficas, Tema 3, Serie C, Oficina de Estadísticas de las Comunidades Europeas, Luxemburgo, 1989.

España es el único de los cuatro países que tiene una fecundidad que permite el reemplazo de las generaciones en el inicio de los ochenta. Francia inicia una recuperación en 1984 y la RFA en 1986. Italia y España continúan en la secular tendencia a la baja. La diferencia fundamental entre estos 
dos países reside en que los niveles iniciales eran muy dispares. La reducción de la fecundidad española ha sido más rápida. No resulta evidente que la recuperación de Francia y la RFA sea permanente. Mientras que Francia se ha estabilizado en 1,82 hijos por mujer en 1987 y 1988, la RFA ha incrementado su índice sintético de fecundidad desde 1986, situándose por encima de Italia (Population, 1989).

\section{TABLA 5}

Edad media de la madre al nacimiento del primer hijo, 1980-1986 (Varios países europeos)

\begin{tabular}{|c|c|c|c|c|c|c|c|}
\hline \multirow[b]{2}{*}{ Paises } & \multicolumn{7}{|c|}{$A \bar{N} O S$} \\
\hline & 1980 & 1981 & 1982 & 1983 & 1984 & 1985 & 1986 \\
\hline España & 24,6 & 24,8 & 24,9 & 25,1 & 25,2 & - & - \\
\hline 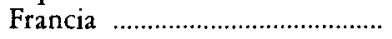 & 24,9 & 25,0 & 25,2 & 25,4 & 25,6 & 25,9 & 26,1 \\
\hline Italia & 24,4 & 24,5 & 24,6 & 24,7 & 24,9 & 25,1 & - \\
\hline RFA & 25,2 & 25,3 & 25,5 & 25,7 & 26,0 & 26,2 & 26,4 \\
\hline
\end{tabular}

NOTA: Los datos de Francia corresponden a la población metropolitana (territorio europeo).

FUENTE: Idem tabla 4 .

Al reducirse la fecundidad se produce un lógico proceso de rejuvenecimiento de la edad media a la maternidad. Una vez que el número medio de hijos por mujer se sitúa próximo a dos, la edad media comienza a incrementarse nuevamente. El aumento de la edad se produce, consecuentemente, con el de la edad media al primer matrimonio. Los jóvenes retrasan tanto el casamiento como la llegada del primer hijo, por lo que ambos efectos empujan al alza la edad media a la maternidad.

En la tabla 5 constan las edades medias de la madre a la llegada del primer hijo. Esta es más baja que la edad media a la maternidad, ya que sólo se ha tenido en cuenta el primogénito. Dado lo reducido de los niveles de fecundidad en los países analizados, la edad en la que la mujer comienza a constituir la descendencia es significativa en sí. La edad media a la llegada del primer hijo también ha aumentado en los últimos años. Ello implica que el intervalo que más se ha incrementado es el protogenésico y no el intergenésico. Este incremento se registra en Italia en 1976. En la RFA, el proceso es anterior por dos razones. Por una parte, la fecundidad se había reducido más tempranamente que en Italia. Por la otra, la edad media había descendido más. A partir de 1972, la edad vuelve a subir. 
Francia y España representan los casos límites, por su temprano y tardío incremento. En el primer país, es en 1971 cuando la edad media vuelve a elevarse. En el segundo, el aumento se registra en 1980. Las edades medias no difieren sustancialmente entre los cuatro países. Italia presenta la más baja y la RFA la más elevada, si comparamos 1984, último año para el cual hay datos para España. La diferencia alcanza un año. A pesar de que los procesos no han sido sincrónicos, las edades no presentan grandes diferencias, ni aun en los momentos en que las tendencias eran contrarias. Ello se debe, en parte, a que España ha tenido una edad media a la maternidad relativamente tardía, respecto a Europa. El lento declive que experimentó la fecundidad hasta 1980 y su elevado nivel incidió en la permanencia en edades relativamente altas.

\section{DISCUSION}

La actividad femenina y la fecundidad se han visto alteradas en la presente década. Los ritmos de cambio han sido diferentes según los países, pero en todos se han registrado hechos que merecen ser analizados. La participación de la mujer en la población activa se ha incrementado en Francia, Italia y RFA entre 1982 y 1984, al contrario que en España, que ha descendido considerablemente. En ese período, el descenso de la fecundidad se ha interrumpido en Francia, para luego estabilizarse, y en la RFA, donde continúa incrementándose. Al contrario, en España e Italia el declive continúa, registrándose niveles muy bajos en estos momentos. La fecundidad actual de estos dos países contrasta con la observada en la década del setenta, que en ambos casos era relativamente elevada, sobre todo comparativamente al resto de países europeos. Tanto en Italia como en España, los ritmos de cambio han sido mucho más rápidos que en los otros países. Los descensos no sólo se han registrado en períodos más cortos de tiempo, sino que también han sido más drásticos.

En este contexto de tendencias contrapuestas, no se puede afirmar que la actividad de la mujer continúe siendo un factor decisivo de la fecundidad. La relación debe ser revisada a la luz de los cambios y alteraciones que se han registrado en la presente década. Uno de los aspectos que deberían ser considerados, cuando se analiza la mutua relación de estas variables, es la categoría socioprofesional de la mujer. Su nivel de instrucción y los motivos que le inducen a trabajar pueden ser más importantes que la dualidad clásica entre maternidad y trabajo.

La inserción de la mujer en la población activa continúa siendo distinta a la del hombre. No se trata de las oportunidades que tienen frente a un empleo, sino al sitio que ocupan, en igualdad de titulación. La población femenina tiene menos posibilidades de independizarse y el proceso de sala- 
rización que afecta a las profesionales es mucho mayor que en el sexo masculino. La mujer, para trabajar, tiene que tener un nivel medio de instrucción superior al del hombre. Mientras que la mayoría de los hombres trabajan, las mujeres lo hacen sobre todo si están en condiciones de conseguir un buen empleo, por su nivel de capacitación para esos cargos.

El hecho de que la población activa femenina esté sesgada implica que las motivaciones para integrarse en ella no son las mismas que en los hombres. Las mujeres fluctúan, entrando y saliendo de la actividad en función de criterios diferentes. Las crisis económicas las afectan en mayor medida. Frente a la penuria de empleo, las mujeres prefieren no buscarlo y volver al hogar. El precio de la sustitución mientras buscan puede ser demasiado elevado y, en consecuencia, permanecen a la espera de un cambio de la situación económica. También por esta razón puede que la población activa femenina esté subestimada, ya que en situaciones críticas las mujeres con menor nivel de capacitación podrían entrar en la economía sumergida.

La mejora en el nivel de instrucción de la mujer en España no se ha traducido en un incremento de su participación en la población activa, sino en un aumento de la edad de entrada. El retroceso en la proporción de mujeres activas registrado en la presente década es indicativo de los condicionamientos a los que la población femenina está sometida. Si la mujer entra y sale por razones coyunturales, difícilmente sus proyectos de constitución de la descendencia dependan de su permanencia en la actividad. La fecundidad y la actividad femenina en España se han reducido drásticamente en el mismo período. Difícilmente se puede establecer una relación inversa entre estas variables. En el corto plazo, las fluctuaciones de cada una de ellas pudieran tener cierta independencia. En el largo plazo, como parte de los proyectos de vida de la mujer, deben establecerse mutuos condicionamientos.

La mujer obrera puede tener una disyuntiva entre trabajar y tener hijos, no por su condición o nivel de instrucción, sino por razones económicas. Su falta en el hogar implica un coste económico que tiene que sufragar. Para ello es necesario que su salario y los costes que implica trabajar fuera cubran estas sustituciones, dejando una cantidad para otros gastos. Dadas las condiciones de trabajo actuales de las personas con bajo nivel de capacitación, es lógico que prefieran permanecer en sus casa, a menos que económicamente les compense salir a trabajar fuera. Esto implica que la elección entre el trabajo remunerado y la sola ocupación de ama de casa no debe depender de los proyectos de vida personales de la mujer, sino del nivel del salario que pudiera ganar.

Las mujeres que trabajan en empleos que requieren una capacitación media se encuentran en una situación diferente. Su salario y sus condiciones de trabajo son mejores que las que corresponden al sector obrero, por lo que es más fácil que permanezcan en sus empleos. Este grupo social es el que menos hijos tiene, en España como en otros países. Pero el coste de 
mantener un cierto status y nivel de vida puede convertir el salario de la mujer en una necesidad familiar, de todo el grupo.

Las mujeres profesionales tienen otras aspiraciones. Su nivel de instrucción y su formación les han llevado a retrasar el casamiento. La llegada del primer hijo puede no cambiar sustancialmente su sistema de vida. Conseguir personas que las reemplacen en el hogar no sólo es más fácil económicamente, sino emocionalmente. Forma parte de su estilo de vida tener ayuda en la casa, por lo que puede que no se presente un conflicto en el momento de la decisión. El hecho de incorporar tempranamente al niño al sistema educativo tampoco proviene de su inserción en el mercado de trabajo, sino que forma parte de su concepción de la crianza. El coste es un problema que no se plantea como central para una familia con altos niveles de ingresos.

El incremento de las madres solteras no implica el retorno a situaciones pasadas de abandono o desahucio. Es una elección, cada vez más libre y consciente, de mujeres que trabajan y tienen un nivel de instrucción superior a la media. La doble carrera ya no es una opción exclusiva de la familia clásica. El modelo descrito por Parsons tiende a desaparecer, no tanto por un simple cambio de roles, sino por una transformación profunda de la estructura del grupo familiar. Las madres solteras constituyen un ejemplo de cómo puede no haber dilema entre maternidad y trabajo.

En este contexto, las medidas políticas que apunten a un abandono del trabajo femenino para el incremento de la descendencia pueden tener resultados muy limitados. Por una parte, habría que considerar a quién van dirigidas. Son las mujeres obreras las que más fácilmente aceptarían esta propuesta, por las mismas motivaciones que las han llevado a tener un empleo. Pero las mujeres que ocupan puestos intermedios o superiores tienen otras razones para trabajar y unos proyectos de vida y familia explícitos, que difícilmente cambiará una simple propuesta económica. 


\section{BIBLIOGRAFIA}

BOUdON, Raymond (1973): L'inegalité des chances, Armand Colin, París.

CADLWELL, John C. (1982): Theory of Fertility Decline, Academic Press, Londres.

Chesnals, Jean-Claude (1986): La Transition démograpbique, PUF, París.

Delgado, Margarita, y FERnÁNDEz CORDón, Juan Antonio (1989): La fecundidad en España desde 1975, Documento de Trabajo núm. 2, Instituto de Demografía, CSIC, Madrid.

Delgado, Margarita (1990): La fecundidad en España por grupos de edad, Serie Documentos de Trabajo, Instituto de Demografía, CSIC, Madrid.

FERNÁNDEZ MÉNDEZ De ANDRÉs, Fernando (dir. del estudio) (1987): Actividad laboral de la mujer en relación a la fecundidad, Ministerio de Cultura, Instituto de la Mujer, Madrid.

INE (1978): Encuesta de Fecundidad. Metodología y resultados (diciembre 1977), Madrid.

- (1986): Encuesta de Fecundidad, 1985, 2 vols., Madrid, diciembre.

INED (1989): "Dix-huitième Rapport sur la situation démographique de la France", Population, núms. 4-5, INED, París, julio-octubre, pp. 711-776.

LEBOUTE, René (1987): "Au carrefour des transitions: fécondité, niveau de vie et culture populaire", Annales de Démographie Historique, Editions de l'Ecole des Hautes Etudes en Sciences Sociales, París, pp. 175-211.

Overbeek, J. (1974): History of Population Theories, Rotterdam University Press, Holanda.

TODOROVIC, Gordana (1985): “Women's Activity and Education as factors of Family size and attitudes towards it in Populations on vatious stages of Demographic Transition", Conferencia de la IUSSP, Florencia, junio, p. 15.

United Nations (1983): "Recents Trends and Conditions of Fertility", Population Bulletin of the United Nations, num. 15, UN, Nueva York. 